\title{
A NEW CONCEPT IN THE DESIGN OF THE LHC BEAM DUMP
}

\author{
J.M. Zazula, M. Gyr, G.R. Stevenson and E. Weisse, \\ European Laboratory for Particle Physics, CERN, CH-1211 Geneva 23
}

\section{Abstract}

Recent Monte Carlo cascade simulations have shown that a reduction in the maximum of deposited energy density can be obtained by applying a uniform magnetic ®eld over a front part of the graphite core of the LHC dump. This paper shows the effect of ®eld strength on spatial distributions of absorbed energy and temperatures, and discusses problems to be solved when designing a suitable magnet.

\section{INTRODUCTION}

The LHC energy of nominally $333 \mathrm{MJ}$ per ring $\left(2.97 \cdot 10^{14}\right.$ protons at 7.0 TeV), extremely concentrated in a small region around the beam axis, gives rise to severe thermal and mechanical constraints on the construction of the beam dump, which will be installed at a distance of about $750 \mathrm{~m}$ from the ejection point. Several beam diluting procedures exist and have already been discussed [1]. Each of them applied alone is either not suf®cient to keep the maximum temperature rise in the graphite below a tolerable levelĐor kicker magnet performance or the tunnel length are forced to extreme levels.

Absorption of the beam energy is the process mainly contributed to by low-energy charged components (mostly electrons and positrons) of the cascades induced by primary protons in the dump. Thus a magnetic ®eld applied over a front part of the core could serve to spread a part of the deposited energy out of the critical concentration region. This study aims to answer what ®eld strengths would be required to obtain a suf®cient cascade dilution, and if those ®elds can be provided by any magnet that is realistic for design and installation in the dump area.

\section{EFFECT OF MAGNETIC FIELD ON ABSORBED ENERGY AND TEMPERATURE DISTRIBUTIONS}

\section{A. Simulations of particle cascades}

The simulations of particle cascades in a central part of the LHC dump were performed with the FLUKA high energy shower program [2]. Comparisons with measurements of absorbed dose distributions around accelerator beams (albeit, at lower energies) have shown [3] that an accuracy of better than $25 \%$ can be expected when estimating the densities of deposited energy from Monte Carlo calculations with this code.

The LHC beam of $7 \mathrm{TeV}$ protons was assumed to have projected distributions of Gaussian pro®le; the beam parameters taken for the simulations are given in Table I. The most suitable material for the construction of the upstream part of the dump core is a graphite, assumed here to be pure ${ }^{12} \mathrm{C}$ of density 1.75 $\mathrm{g} \cdot \mathrm{cm}^{-3}$. Secondary cascades were simulated only in the central part of the dump of dimensions $10 \times 10 \times 300 \mathrm{~cm}^{3}$; preliminary results have shown that this depth includes the longitudi-
Table I

Nominal beam parameters assumed for the simulations.

\begin{tabular}{|l|rl|}
\hline Beam momentum (monoenergetic) & 7.0 & $\mathrm{TeV} / c$ \\
Horizontal beam size (Gaussian $\sigma_{h}$ ) & 1.46 & $\mathrm{~mm}$ \\
Vertical beam size (Gaussian $\sigma_{v}$ ) & 1.06 & $\mathrm{~mm}$ \\
Beam divergence (Gaussian $\sigma_{h, v}$ ) & 1.64 & $\mu \mathrm{r}$ \\
Beam intensity (protons per ring) & 2.97 & $\cdot 10^{14}$ \\
Spill absorption time & 90 & $\mu \mathrm{s}$ \\
\hline
\end{tabular}

nal maxima of deposited energy density (with or without a magnetic $® e l d$ ), and that the energy densities (and the corresponding instantaneous temperature rises) decrease by at least two orders of magnitude for radial positions $5 \mathrm{~cm}$ from the beam axis.

The interactions and propagation of charged components of the cascades (protons, charged pions, muons and electrons) were followed down to the kinetic energy threshold of $1 \mathrm{MeV}$, of photons down to $100 \mathrm{keV}$, and of neutrons down to a thermal energy range. Particles slowed down or produced with energies below these thresholds are assumed to deposit their energy locally (in a range which is negligible compared to the scoring mesh size). Energy lost by charged particles in ionization processes was converted to emitted $\delta$-rays (low energy electrons), and thus further distributed around ionizing particle tracks. None of the biasing or importance sampling methods available in FLUKA were used.

The homogeneous uni-directional magnetic ®eld perpendicular to the beam axis (the direction of ®eld is called ${ }^{\mathrm{a}} \mathrm{vertical}^{\circ}$, since the induction $\vec{B}$ was taken parallel to the vertical beam plane) was present over the whole length of the system $(3 \mathrm{~m})$, with ®eld strengths of $0.5,1,2$ and $5 \mathrm{~T}$. The results without $®$ eld were also obtained for reference, for the same beam (see Table I) and for the beam that vertical pro®le was linearly swept over distance $\pm 5 \mathrm{~cm}$. Samples of primary histories that were completed for $® x e d$ run times of the simulation program (20 000 native seconds of the SP2 system at CERN) for each ®eld case are given in the the second column of Table II.

The density of the deposited energy was determined as a function of horizontal and vertical position and of longitudinal depth, in two Cartesian bin structures, i.e.,: a $®$ ne mesh of $0.1 \times 0.1 \mathrm{~mm}^{2}$ lateral size (up to $5 \mathrm{~mm}$ from the beam axis), and a coarse mesh of $1 \times 1 \mathrm{~mm}^{2}$ lateral size; both with longitudinal bins $10 \mathrm{~cm}$ in depth.

\section{B. Spatial distributions of deposited energy}

Maximum energy densities, maximum laterally integrated energies and total energies deposited per one proton of the LHC beam, in the $10 \times 10 \times 300 \mathrm{~cm}^{3}$ graphite block in an uniform magnetic ®elds of various strengths, are given in Table II. The maximum densities given in the third column of the table, are those 


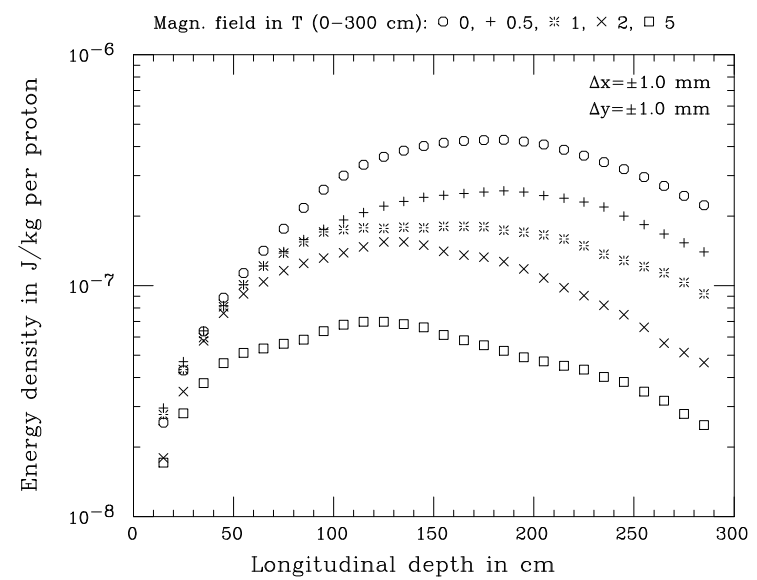

Figure 1. Maximum energy densities as function of longitudinal depth in the core, for various ${ }^{\circledR}$ eld strengths.

obtained from the $1 \times 1 \mathrm{~mm}^{2}$ lateral bins (coarse mesh), that were still less then projected half-widths of the beam.

The obtained spatial distributions of densities of energy deposited per one primary proton, for various levels of the magnetic ${ }^{\circledR} e l d$, are shown in Figure 1 as a function of the longitudinal depth in graphite, and in Figure 2 as a function of the lateral distance off-axis in the plane perpendicular to the magnetic induction $\vec{B}$ (horizontal plane). For the longitudinal plots, the densities have been averaged over $\pm 1 \mathrm{~mm}$ distances in both lateral planes. The horizontal distributions shown in Figure 2 have been averaged between the symmetrical ${ }^{\mathrm{a}} \mathrm{up}^{\mathrm{o}}$ and ${ }^{\mathrm{a}} \mathrm{down}^{\mathrm{o}}$ positions within the $\pm 1 \mathrm{~mm}$ range in the vertical plane (parallel to $\vec{B}$ ), and over $\pm 30 \mathrm{~cm}$ ranges around the longitudinal maxima respective to the ${ }^{\circledR}$ eld levels, taken from Figure 1, that are: 160$220 \mathrm{~cm}$ for no ${ }^{\circledR}$ eld case, $150-210 \mathrm{~cm}$ for $0.5 \mathrm{~T}, 140-200 \mathrm{~cm}$ for $1 \mathrm{~T}, 100-160 \mathrm{~cm}$ for $2 \mathrm{~T}$, and $80-140 \mathrm{~cm}$ for $5 \mathrm{~T}$. Moreover, the performed analysis has proved that (within the limits of statistical errors) also the ${ }^{a}$ right $^{\circ}$ and ${ }^{a}$ left $^{\circ}$ distributions are symmetrical, and thus the mean values of the ${ }^{\mathrm{a}} \mathrm{right}^{\mathrm{o}}$ and ${ }^{\mathrm{a}} \mathrm{left} \mathrm{t}^{\mathrm{o}}$ horizontal positions (from 0 to $5 \mathrm{~cm}$ ) are plotted in Figure 2.

The ${ }^{\circledR}$ ne mesh results included in Figure 2 (leftmost part of the scale) show again that the horizontal pro®les of energy density, at depths of the longitudinal maxima, are ${ }^{-}$at within the horizontal range of ${ }^{\circledR} \mathrm{rst} \pm 1 \mathrm{~mm}$ off the beam axis. The central parts of the vertical distributions (not shown here to keep this paper concise) look similar. This justi®es again the averaging procedures that were applied for the Figure 1 and Table II.

\section{Maximum temperatures after absorption of the beam}

The amount of beam energy that is deposited in the core is further dissipated in the dump in the form of heat. In order to determine maximum temperature rises that can be expected in the graphite after absorption of the spill, the energy deposition results from particle cascade simulations were coupled to a transient heat transfer analysis, performed by means of the ANSYS (Bnite element program [4]. The system geometry and loads being symmetrical, only one quarter of the core was considered, up to $5 \mathrm{~cm}$ radius off beam axis. This three-dimensional quadrant

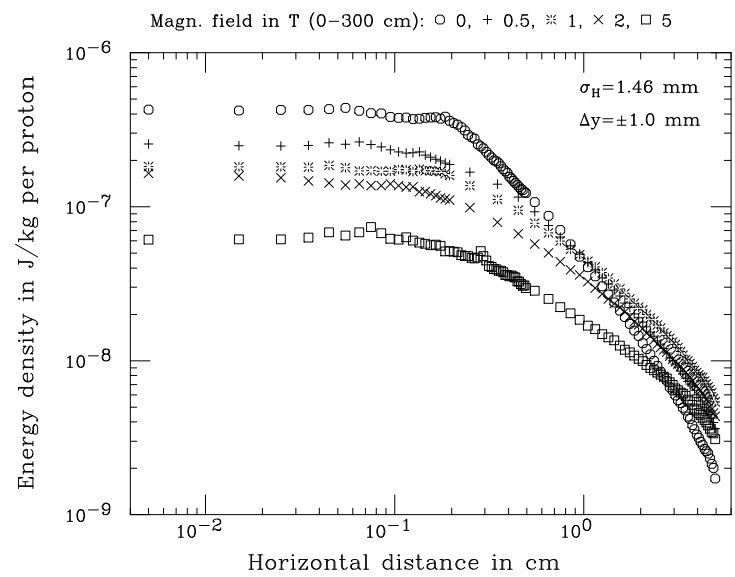

Figure 2. Lateral distributions of energy density at depths of longitudinal maxima, for various ®eld strengths.

Table II

Total energies deposited in the core, maximum laterally integrated energies, maximum energy densities and maximum temperature rises for various ®eld strengths.

\begin{tabular}{|c|c||c|c|c|c|}
\hline $\begin{array}{c}\text { Magn. } \\
\text { ßeld } \\
|\vec{B}| \\
\text { in T }\end{array}$ & $\begin{array}{c}\text { No. } \\
\text { of } \\
\text { sim. } \\
\mathrm{p}\end{array}$ & $\begin{array}{c}\text { Depos. } \\
\text { part } \\
\text { of } \\
7 \mathrm{TeV}\end{array}$ & $\begin{array}{c}\text { Energy } \\
\text { density } \\
\text { in } 10^{-7} \\
\mathrm{~J} / \mathrm{kg}^{a}\end{array}$ & $\begin{array}{c}\text { Lateral } \\
\text { integral } \\
\text { in } 10^{-9} \\
\mathrm{~J} / \mathrm{cm}^{a}\end{array}$ & $\begin{array}{c}\text { Temp. } \\
\text { rise } \\
\text { in } 10^{3} \\
\mathrm{~K}^{b}\end{array}$ \\
\hline \hline no & 71 & $28 \%$ & 5.2 & 1.9 & 51 \\
\hline 0.5 & 57 & $21 \%$ & 3.2 & 1.5 & 33 \\
1.0 & 26 & $19 \%$ & 2.4 & 1.2 & 22 \\
2.0 & 35 & $14 \%$ & 1.9 & 0.8 & 18 \\
5.0 & 45 & $9 \%$ & 0.8 & 0.5 & 9 \\
\hline sweep $^{c}$ & 32 & $24 \%$ & 0.4 & 1.6 & 5 \\
\hline
\end{tabular}

${ }^{a}$ per one primary proton

${ }^{b}$ per spill (2.97·10 $10^{14}$ protons)

${ }^{c} \pm 5 \mathrm{~cm}$, without magnetic ®eld

was meshed with quadrilateral brick elements, by using uniform divisions in the azimuthal angle $\left(\Delta \phi=15^{\circ}\right)$, radial bins increasing logarithmically from $\Delta \mathrm{r}=0.5 \mathrm{~mm}$, and the same longitudinal bins as in the Monte Carlo calculations $(\Delta \mathrm{z}=10 \mathrm{~cm})$. As this mesh is more ef®cient in covering spatial regions of concentrated importance than the Cartesian bins available in FLUKA, much less elements (3600 in total) were required. However, a special interface algorithm had to be written, transferring the Monte Carlo scoring output to the thermal load input of ANSYS.

These loads were the internal heat generation rates (in $\mathrm{J} /\left(\mathrm{cm}^{3} \cdot \mathrm{s}\right)$ ), obtained by multiplying the energy density (in $\mathrm{J} / \mathrm{cm}^{3}$ per primary) interpolated for each node by the number of protons in spill, and dividing them by the spill absorption time in seconds (see Table I). These heat generation rates were assumed to be constant in time during the absorption period, which is long when compared with the time scale of the cascade development, but short when compared to characteristic times of heat propaga- 
tion. Thus heat ${ }^{-}$ow outside of the core (to external parts of the dump, a cooling system, etc.) could be neglected for the absorption period, and the external boundaries of the considered system could then be assumed to be adiabatic. Moreover, the thermal properties of graphite, taken from Ref. [5], change considerably with temperature; in particular the speci®c heat varies from about 660 to $2500 \mathrm{~J} /\left(\mathrm{kg} \cdot{ }^{\circ}\right)$ between room temperature and few thousands degrees. Thus ANSYS procedures for the nonlinear solutions had to be involved. It should be noted that physical properties can also vary between different graphites, and under irradiation conditions.

The maximum temperatures at the end of a spill, obtained for each ®eld strength, are given in the last column of Table II. The three-dimensional temperature distributions are shown in the form of color contours in the poster session of this conference.

\section{PROBLEMS TO BE SOLVED BY DESIGNING A SUITABLE MAGNET}

The major dif®culty is the building of a suitable magnet with the necessary high dipole $®$ eld over a $70 \mathrm{~cm}$ wide gap and within a volume of about $1.5 \mathrm{~m}^{3}$, even if the precision of the $囚$ eld is not a critical parameter. By far the most elegant solution, requiring neither power supply, nor water cooling or cryogenics in an area which is radioactive and distant from any central infrastructure, would be a permanent box- or ring-magnet [6] which, because of the very strong magnetic forces (several tons $/ \mathrm{m}$ ) between the different blocks, would be built of short modules which would need to be preassembled. However, the maximum ${ }^{\circledR}$ elds which can be obtained with a ${ }^{\circ}$ reasonable ${ }^{\circ}$ design Đabout $0.3 \mathrm{~T}$ with relatively cheap ferrite, or $1 \mathrm{~T}$ when using the more expensive rare earth - cobalt material \pm are by far insuf®cient to reduce the energy deposition by an order of magnitude. In order to achieve higher $®$ elds, the volume of the permanent magnet material would have to grow out of any proportion. The solution of a classical window-frame electro-magnet cannot be envisaged since the maximum achievable ®eld of $2 \mathrm{~T}$ only brings about one third of the desired effect. The stored magnetic energy in such a magnet would be in the order of $1 \mathrm{MJ} / \mathrm{m}$ when excited with a total current of about $1.2 \cdot 10^{6}$ Ampere-turns. The dissipated power in the aluminum coil, which needs to be cast in concrete, would exceed $1 \mathrm{MW}$ unless being cooled with liquid nitrogen. The only possible magnet able to produce the required magnetic ®eld of $5 \mathrm{~T}$ would be a superconducting magnet which provides a stored magnetic energy of about $5 \mathrm{MJ} / \mathrm{m}$ and which possibly would quench at every discharge of the LHC-beam. The coil of the magnet would have to be fully stabilized in order to absorb the total energy and the helium be contained in a closed circuit.

\section{CONCLUSIONS}

This study of the utility of an uniform magnetic ®eld for the LHC beam dump system can be summarized:

- The maximum energy densities and temperatures are reduced approximately linearly with ®eld strength, up to about factor of 7 for a $5 \mathrm{~T}$ 囚eld. The effect obtained for the maximum @eld is comparable with the dilution of energy density obtained with a linear sweep of $\pm 5 \mathrm{~cm}$.
- In presence of the magnetic $® e l d$, spatial pro®les of deposited energy are longitudinally -attened and laterally spread out from the central region; thus smaller fractions of the total energy are deposited in the central part of the dump system, critical for the concentration of the absorbed energy and consequent temperature rise;

- It was not possible to obtain a reduction of the maximum energy density by one order of magnitude, or to keep the maximum temperatures in graphite below $2500^{\circ} \mathrm{C}$, using the ®eld strengths provided by the most favorable permanent magnets; other magnet designs would be even less realistic. A satisfactory solution might be achieved be combining magnetic dilution of the cascades with a linear sweep in the perpendicular direction.

\section{References}

[1] A.Ferrari, G.R.Stevenson and E.Weisse, a Design of the

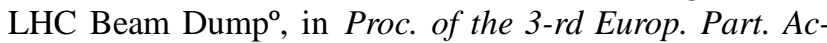
cel. Conf. (Vol. 2, pp. 1545-47), Berlin (March 24-28 1992); LHC Note 180.

[2] A. Fassó A. Ferrari, J. Ranft and P.R. Sala, 'FLUKA: Present status and future developments ${ }^{\mathbf{0}}$, in Proc. of the IV Int. Conf. on Calorimetry in High Energy Physics (p. 493), La Biodola, Italy (Sept. 20-25, 1993).

[3] A. Fassó A. Ferrari, J. Ranft, P.R. Sala, G.R. Stevenson and J. M. Zazula, ${ }^{a}$ Comparison of FLUKA simulations with measurements of ${ }^{-}$uence and dose in calorimeter structures $^{\circ}$, Nucl. Instr. and Meth. A 332 (1993) 459-468.

[4] Swanson Analysis Systems, Inc., ANSYS (Revision 5.1), SASI/DN-P511:51, Houston, USA (Sept. 30 1994).

[5] B.T. Kelly, ${ }^{a}$ Physics of graphite ${ }^{o}$, Appl. Sci. Publ., LTD (London 1981).

[6] K. Halbach, ${ }^{\mathrm{a}}$ Application of permanent magnets in accelerators and electron storage rings ${ }^{\circ}$, Journ. of Appl. Phys. 57-8, part IIA (1985) 3605-08. 